\title{
Determination of the temperature dependence of tungsten erosion
}

\author{
H. Maier ${ }^{*}$, H. Greuner, U. v. Toussaint, M. Balden, B. Böswirth, S. Elgeti \\ Max-Planck-Institut für Plasmaphysik, Boltzmannstr. 2, 85748 Garching, Germany
}

We present the results of erosion measurements on actively cooled tungsten samples at quasiconstant surface temperature conditions performed in the high heat flux facility GLADIS.The samples were exposed to a $\mathrm{H}$ beam at a central power density of $10 \mathrm{MW} / \mathrm{m}^{2}$ up to a fluence of $10^{26} \mathrm{~m}^{-2}$. We observe a weak temperature dependence of the erosion yield. The data are compared with similar data obtained from loading with a $\mathrm{H}$ beam with $\mathrm{He}$ admixture. Both datasets are analysed in a probabilistic approach. We obtain activation energies of $0.04 \mathrm{eV}$ and $0.06 \mathrm{eV}$ for the cases with and without $\mathrm{He}$, respectively.

\section{Corresponding Author Address:}

Hans Maier, Max-Planck-Institut für Plasmaphysik, Boltzmannstr. 2, 85748 Garching, Germany

Corresponding Author e-mail: Hans.Maier@ipp.mpg.de 


\section{Introduction}

Tungsten is considered to be the most viable armour material for the plasma-facing components of a fusion reactor, especially for divertor components [1, 2, 3]. The most important reason for this is the long lifetime of tungsten under erosion by physical sputtering, which is due to its high atomic mass and high surface binding energy. In the literature it is usually assumed that physical sputtering is a process, which is insensitive to the target temperature, see e. g. [4]. Temperature dependent erosion yields of liquid and solid metals have, however, been reported in the past, e.g. [5, 6, 7], and also very recently [8]. As the sputtering process is based on binary collisional energy transfer there should not be any principal difference between solids and liquids in this respect.

The behaviour of tungsten materials under a variety of heat and particle loads has been investigated in various devices, e. g. $[9,10,11,12,13]$. In the special case of He impingement on tungsten it is well known that this leads to the formation of complex surface morphologies (see the review [14] and also [15]).

Using our high heat flux facility GLADIS [16] we investigate actively water-cooled tungsten samples under high heat flux loading with $\mathrm{H} / \mathrm{He}$ mixed neutral beams at quasi-stationary surface temperatures. We have recently devised a method to measure erosion in the $\mu \mathrm{m}$ range on such samples [17] and a thorough data analysis revealed an Arrhenius-like temperature dependence in the case of loading with a $\mathrm{H}$ beam with a 6 atomic percent $\mathrm{He}$ admixture [18]. A temperature dependent erosion behaviour was reported recently in the case of bombarding tungsten with a He plasma [9], which would in principle be in line with our findings cited above. The results even indicated erosion below the sputtering threshold energy. However, high temperature tungsten erosion measurements in the TEXTOR edge plasma were well explicable by a combination of temperature independent physical sputtering by carbon impurities and thermal sublimation [19]. No further enhancement of atomic tungsten release 
was observed up to $3600 \mathrm{~K}$. Therefore one might speculate that the presence of $\mathrm{He}$ and/or its influence on the morphology formation has an influence on the reported temperature dependences in [9] and [18]. For this reason we have employed our erosion measurement method to investigate the case of loading with a pure hydrogen beam.

In this contribution we present a comparison of the new experimental results on loading with pure $\mathrm{H}$ with the published results on loading with a $\mathrm{H} / 6 \% \mathrm{He}$ mixture. We present a thorough data analysis based on maximum-likelihood considerations (see e. g. [20]) with error estimates. Finally we extend our maximum likelihood considerations to compute the full probability distribution for the activation energy of the temperature dependent erosion data.

\section{Experiment and results}

The experiments were performed on actively water cooled samples under quasi-stationary surface temperature conditions in the high heat flux test facility GLADIS, which is described in [16]. Commercially available tungsten from Plansee SE with a purity of $99.97 \%$ was employed to manufacture the samples. The surfaces were mechanically polished. The design of the employed mockup as well as the sample preparation and analysis was identical to the procedures described in detail in [17]: For the erosion measurements markers are engraved into the side of the samples by focussed ion beam milling. The distance of these markers to the beam-exposed surface of the respective sample is measured by electron microscopy before and after exposure. The difference of these two distance measurements gives the amount of eroded material. This procedure results typically in a precision of $100 \mathrm{~nm}$ to $1 \mu \mathrm{m}$, depending on the amount of erosion. During the erosion process additionally a roughening of the surfaces occurs. This is mainly caused by the grain orientation dependence of physical sputtering and increases with fluence. Since our erosion measurement is local this effect 
introduces an additional uncertainty. This is taken into account by measuring the surface topography using confocal scanning laser microscopy. The mean square deviations from the average height level are then used as additional error for the individual erosion data points. For most data points this is the dominating contribution to our error estimate.

The surface temperatures of the samples ranged from $600^{\circ} \mathrm{C}$ to $2000^{\circ} \mathrm{C}$. The samples were exposed to a neutral hydrogen beam with a Gaussian beam profile at a central power density of $10.5 \mathrm{MW} / \mathrm{m}^{2}$ with individual pulses lasting $30 \mathrm{~s}$ each. The local flux varies between 3.7 and $4.1 \times 10^{21} \mathrm{H} / \mathrm{m}^{2} \mathrm{~s}$. The acceleration voltage was $29 \mathrm{kV}$ resulting in an average particle energy of $16 \mathrm{keV}$ per atom, see e.g. [17]

Samples were removed after total fluences of $1 \times 10^{25} \mathrm{H} / \mathrm{m}^{2}$ and $1 \times 10^{26} \mathrm{H} / \mathrm{m}^{2}$, respectively. Depending on the individual position of the respective sample the above fluences have to be scaled with 0.98 for $600^{\circ} \mathrm{C}, 1.03$ for $1000^{\circ} \mathrm{C}$ and $1500^{\circ} \mathrm{C}$ and 1.08 for $2000^{\circ} \mathrm{C}$.

The results of the erosion measurements are given in figure 1 together with the total error estimate. The low temperature data are consistent with published physical sputtering yields $[21]$.

Figure 2 shows a comparison of cross-sections prepared by focussed ion beam (FIB) from samples loaded at a surface temperature of $2000^{\circ} \mathrm{C}$ with pure $\mathrm{H}$ (top) and $\mathrm{H} / 6 \% \mathrm{He}$ (bottom), respectively. The figure clearly shows that the pronounced morphology formation, which is observed in the presence of $\mathrm{He}$, is absent in the case of irradiation with pure $\mathrm{H}$. The presence of $\mathrm{He}$ in the GLADIS beam causes a complex three-dimensional surface structure (see $[17,18]$ ), which yields a strong contribution to the surface roughness contributing to the uncertainty mentioned above. In addition the bottom sample shows a bubble-rich layer extending nearly half a micron below the surface. There are bubbles visible in the subsurface cross-section in the hydrogen case as well, however on a comparably sporadic level. 


\section{Data analysis and discussion}

\subsection{Erosion yields}

As already pointed out in [18] a careful choice of the analysis method is required in an analysis based on very few data points the quality of which is unknown. If a measurement yields a large number of data points, outliers are easily identified and can be treated e. g. by median filtering. Our situation, however, is the opposite.

Since the usual least squares analysis scheme is rather sensitive to outlier data points because of their large contribution, we chose a "robust estimation" method: We minimize the sum of the absolute deviations instead of the squares to determine the model parameter which maximises the likelihood of our data. The concept of robust estimation is described in [20].

The model we want to use to describe our data is:

$$
Y=\boldsymbol{a} \cdot X
$$

Here $X$ is the fluence, $Y$ the amount of erosion and $\boldsymbol{a}$ represents the erosion yield. To find the maximum likelihood erosion yield $\boldsymbol{a}$ we determine

$$
\min _{\boldsymbol{a}} \sum_{i} \frac{\left|Y_{i}-\boldsymbol{a} \cdot X_{i}\right|}{\sigma_{i}}
$$

Here $Y_{i}$ are the individual erosion values at fluences $X_{i}$ and $\sigma_{i}$ are the corresponding error estimates. This procedure yields a value of the erosion yield for each of the investigated sample surface temperatures. This kind of analysis was performed for the present case of loading with a pure $\mathrm{H}$ beam. In [18] the same kind of analysis was described for the case of loading with a $\mathrm{H}$ beam containing $6 \%$ of $\mathrm{He}$ admixture. Figure 3 shows the erosion yields 
determined for both cases as a function of the sample surface temperature. The error bars in the figure are set such that they correspond to the usual $1 \sigma$ standard deviation error of Gaussian statistics, i. e. they cover a $68 \%$ probability interval.

In Figure 3 it can be seen that both data sets show a trend for the erosion yield to increase with temperature. The trend, however, barely exceeds the error margins. So the question arises whether or how definitely the data really represent the presence of a temperature dependent mechanism in the erosion process. In the case of a thermally activated process with a Boltzmann-like temperature dependence the traditional way is to analyse the data by performing a linear fit in an Arrhenius representation as shown in [18]. Here we want to proceed instead with a probabilistic approach, which is presented in the next section.

\subsection{Activation energy}

The procedure described above yields maximum likelihood values for the erosion yield at each temperature. An extension of the above erosion model equation (1) to a temperature dependent model can be performed by introducing a temperature dependent erosion yield:

$$
\boldsymbol{a}=a_{0} \times \exp \left(-\frac{E}{k T}\right)
$$

with $a_{0}$ the prefactor and $E$ the activation energy of the erosion yield. In such a model the question whether or not the described process is temperature dependent is translated into the question what is the probability of a non-zero activation energy. Therefore now we are not only interested in a maximum likelihood activation energy $E$, but instead in the whole probability distribution $p\left(E, a_{0}\right)$. Extending the considerations correspondingly we now investigate the probability distribution: 


$$
p\left(E, a_{0}\right)=\frac{1}{Z} \exp \left(-\sum_{i}\left|Y_{i}-\boldsymbol{a} X_{i}\right| / \sigma_{i}\right)=\frac{1}{Z} \prod_{i} \exp \left[-\frac{\left|Y_{i}-\boldsymbol{a} X_{i}\right|}{\sigma_{i}}\right]
$$

with $\boldsymbol{a}$ given by equation (3). Here the last equality reflects the fact that all individual measurement data points are independent of each other. $\mathrm{Z}$ is the normalisation constant.

Equation (4) can be evaluated for both of our datasets individually to yield the probability distribution. This is shown in figure 4. Both distributions show a clear maximum with respect to the two variables. In the present discussion we are, however, only interested in the probabilities of the activation energies. We can generate univariate distributions by integrating one dimension:

$$
P(E)=\int p\left(E, a_{0}\right) d a_{0}
$$

The resulting probability densities are plotted in figure 5. If we use the maximum and error bars corresponding to the usual $1 \sigma$ standard deviation error of Gaussian statistics, i. e. covering the $68 \%$ probability interval, we obtain:

$$
E=0.04_{-0.02}^{+0.02} \mathrm{eV}\left(\mathrm{H} \mathrm{w.} \mathrm{6 \%} \mathrm{He)} \quad E=0.06_{-0.02}^{+0.07} \mathrm{eV}(\text { pure H) }\right.
$$

The first of these two reproduces the results obtained from the Arrhenius analysis presented in [18]. Within the error margins the two values coincide. This means that the presence of $\mathrm{He}$ and its influence on the morphology do not affect the activation energy significantly.

By setting a limit for the activation energy we are now able to make a quantitative probabilistic statement as to whether or not our data contain a thermally activated process. As mentioned before, the presence of a temperature dependence in our model as specified by equations (1) and (3) is reflected by a non-zero activation energy. To obtain a finite probability from the probability density $P(E)$ we have to integrate $P(E)$ over a finite interval. If we set an arbitrary limit of $10 \mathrm{meV}$, we can say that our data analysis yields a probability 
for the activation energy to be above this limit of $97 \%$ in both cases. If we assume $E$ to be identical in both cases and analyse them as one common dataset this probability can even be increased to $98.5 \%$

The activation energy we obtain from our probabilistic data analysis yields a value which lies in an order of magnitude typical for phonons in tungsten [22]. The thermally driven vibrational motion of the atoms in the solid could be a possible source for the temperature dependence we observe. Typically it is argued that the maximum of the transferable energy in a binary collision is given by $E_{\text {transfer }}=E_{0} 4 m_{1} m_{2} /\left(m_{1}+m_{2}\right)^{2}$ and that any contributions due to thermal energies of the target atoms are negligible compared to the projectile energy $E_{0}$. However, a closer look into the physics of binary collisions [23] reveals that the energy transfer to a moving target atom can be higher in the case of target atoms not being at rest. The corrections depend on the mass ratio of target atom to projectile $\left(\mathrm{m}_{2} / \mathrm{m}_{1}\right)$ - i. e. they are most effective for a light projectile and heavy target atoms - and on the ratio of velocities of the collision partners.

It is also known that the presence of $\mathrm{H}$ on the tungsten (110) surface causes a significant modification of the electronic states, which in turn causes an anomaly in the surface phonon dispersion [24]. Vibrational effects of the surface atoms have already been speculated to be the origin of a temperature dependent sputtering yield in [7].

\section{Conclusion}

In our neutral beam high heat flux facility GLADIS we investigated the erosion of tungsten samples during loading at $10.5 \mathrm{MW} / \mathrm{m}^{2}$ central power density at quasi-stationary surface temperatures. In addition to the published temperature dependence of tungsten erosion under loading with a $\mathrm{H}$ beam with $6 \% \mathrm{He}$ admixture we observe also a temperature dependence under loading with pure $\mathrm{H}$. The presence of $\mathrm{He}$ in the beam and its effects on the morphology 
can therefore be ruled out as the sole source for observing a temperature dependent erosion yield.

By performing a probabilistic data analysis based on maximum likelihood considerations and assuming the temperature dependence to be Boltzmann-like, we obtain a probability of $97 \%$ for the activation energy to be above $10 \mathrm{meV}$, which means that the presence of a temperature dependence in our data is clearly significant. We obtain a value for the activation energy of the process of $(0.04 \pm 0.02) \mathrm{eV}$ for a $\mathrm{H}$ beam with $6 \% \mathrm{He}$ and $0.06_{-0.03}^{+0.07} \mathrm{eV}$ for pure $\mathrm{H}$.

\section{Acknowledgement}

This work has been carried out within the framework of the EUROfusion Consortium and has received funding from the European Union's Horizon 2020 research and innovation programme under grant agreement number 633053. The views and opinions expressed herein do not necessarily reflect those of the European Commission. 
Figures:

Figure 1: Experimental results of erosion with pure $\mathrm{H}$ for the four different temperatures and the two different fluence steps. The exact fluence values are given in the text. The error bars are total errors including also the surface roughening (see text).

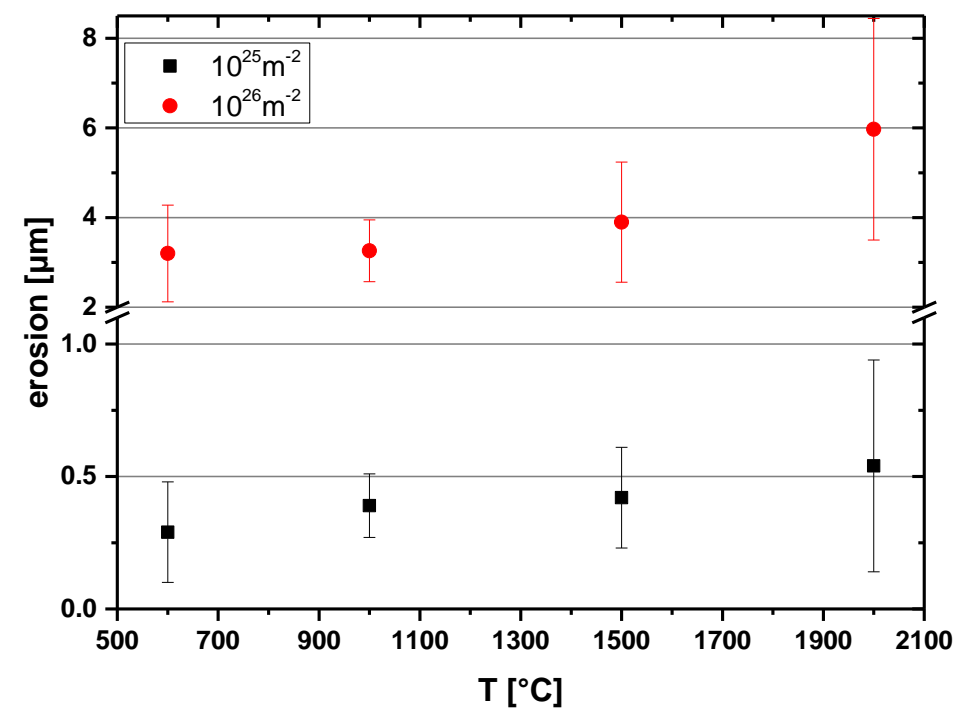


Figure 2: FIB cross sections from samples loaded at a surface temperature of $2000^{\circ} \mathrm{C}$ with a fluence of approximately $1 \times 10^{26} \mathrm{~m}^{-2}$ with a pure $\mathrm{H}$ beam (top) and $\mathrm{H}$ with $6 \% \mathrm{He}$ (bottom, see [18]). The vertical structures below the pores are due to sample preparation (curtaining effect).
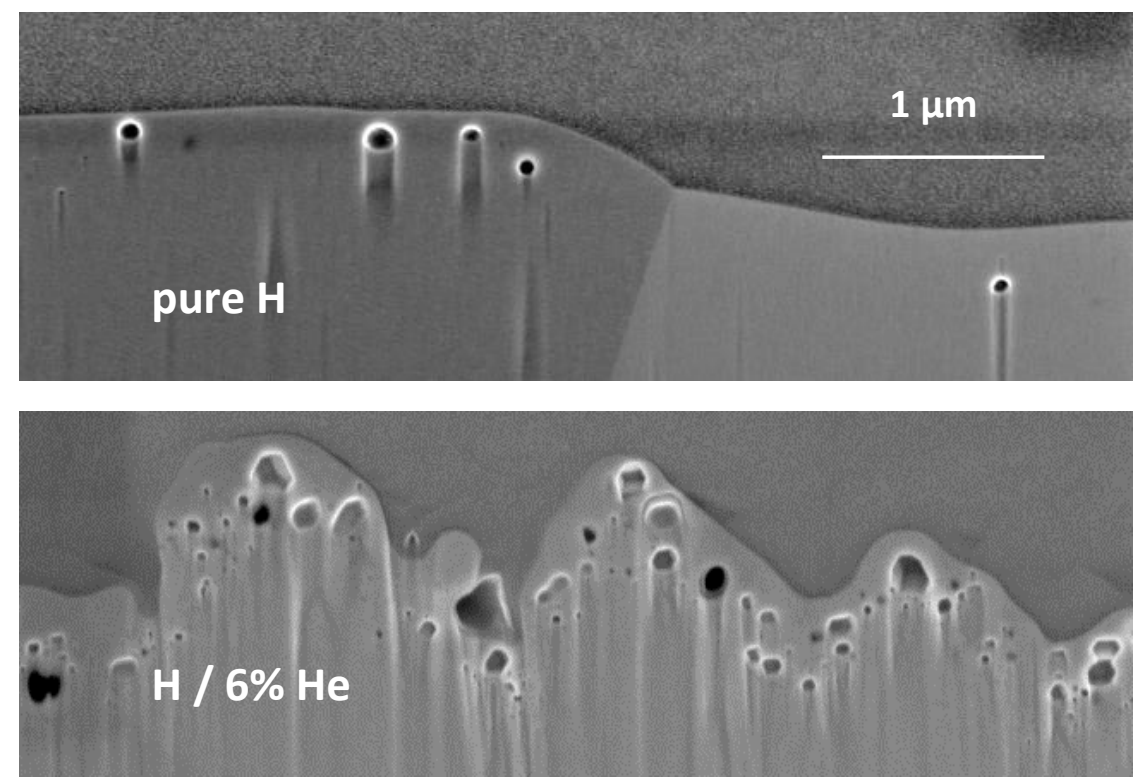
Figure 3: Erosion yields as a function of temperature for samples loaded with a pure $\mathrm{H}$ beam (circles, bottom) and from the data presented in ref $[17,18]$ for loading with a $\mathrm{H}$ beam with $6 \% \mathrm{He}$ admixture (squares, top).

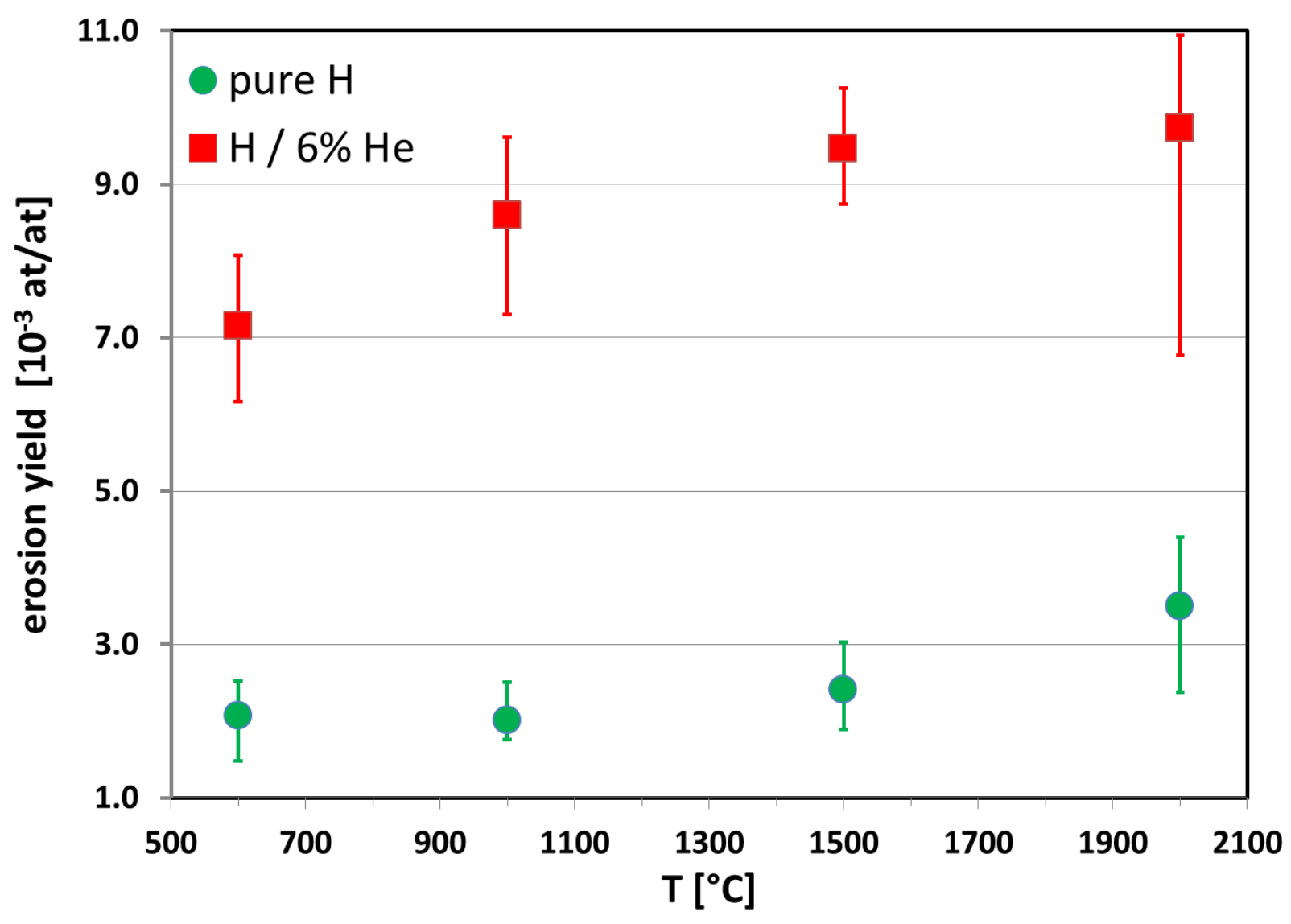


Figure 4: Probability distributions $p\left(E, a_{0}\right)$ computed from the two individual datasets for pure $\mathrm{H}$ erosion as well as for the case of $6 \% \mathrm{He}$ admixture.

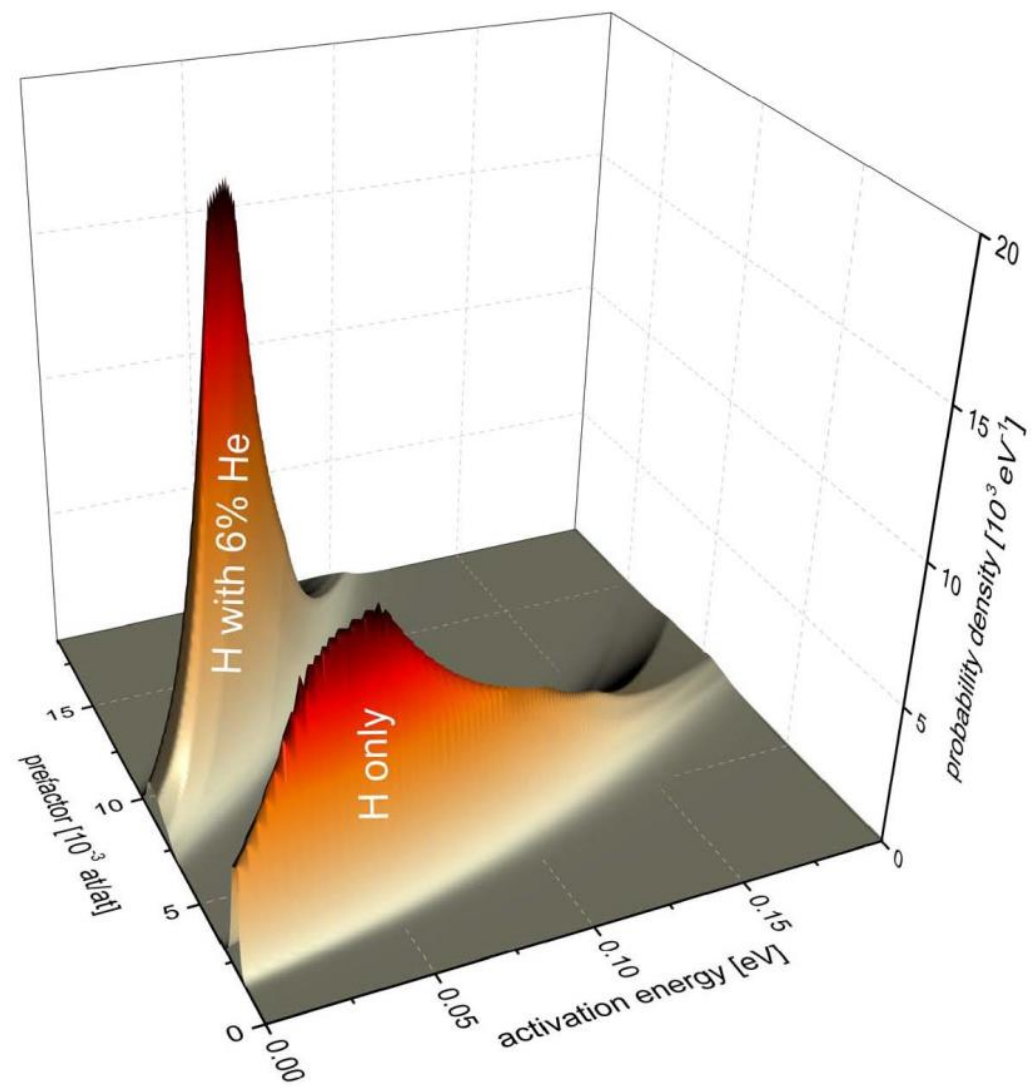


Figure 5: Probability densities $P(E)$ for the two individual data evaluations: solid line: pure $\mathrm{H}$, dashed $\mathrm{H} / 6 \% \mathrm{He}$ (see text).

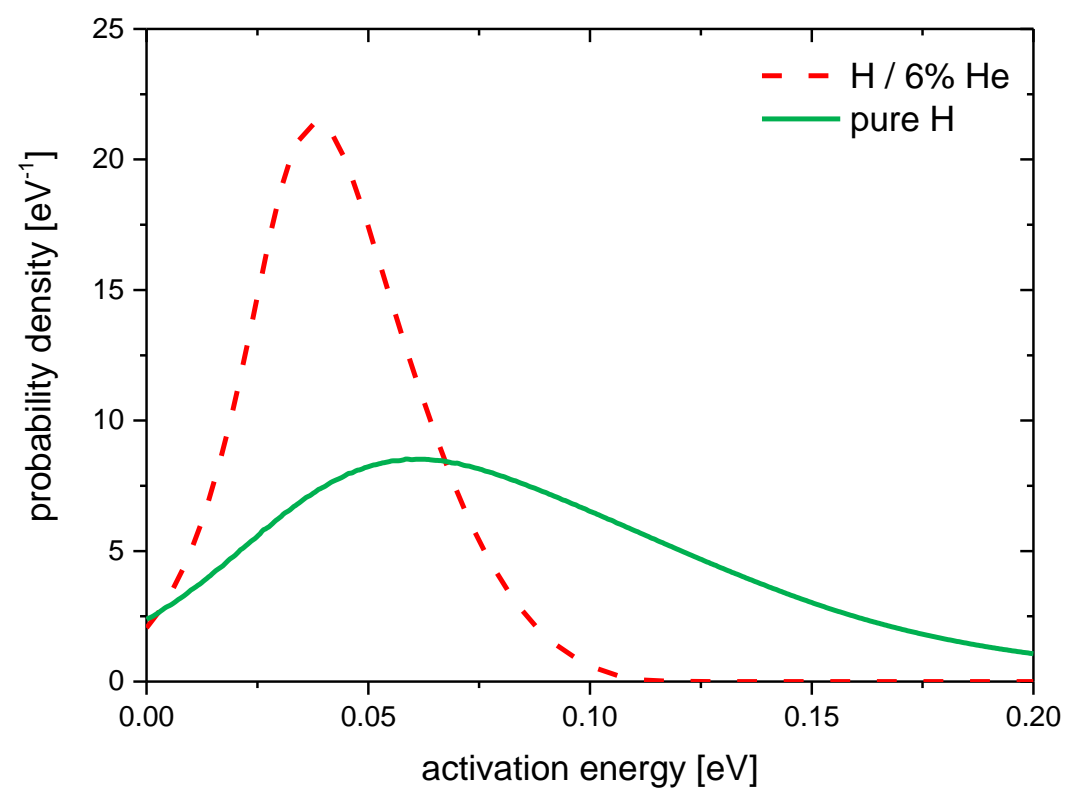




\section{References:}

1 V. Philipps, J. Nucl. Mater. 415 (2011) S2

2 R. Neu et al., Plasma Phys. Contr. Fusion 53 (2011) 124040

3 H. Bolt et al., J. Nucl. Mater. 307-311 (2002) 43

4 P. Sigmund and M. Szymonski, Appl. Phys. A 33 (1984) 141

5 R. P. Doerner et al., J. Nucl. Mater. 313-316 (2003) 383

6 R. W. Conn et al., Nucl. Fusion 42 (2002) 1060

7 C. E. Carlston et al., Phys. Rev. 138 (1965) 759

8 J. W. Coenen et al., Phys. Scr. T159 (2014) 014037

9 G. de Temmerman et al., J. Nucl. Mater. 438 (2013) S78

10 G. Pintsuk et al., J. Nucl. Mater. 438 (2013) S945

11 H. Greuner et al., J. Nucl. Mater. 442 (2013) S256

12 K. R. Umstadter et al., Phys. Scr. T138 (2009) 014047

13 K. Tokunaga et al J. Nucl. Mater. 329-333 (2004) 757

14 Y. Ueda et al., J. Nucl. Mater. 442 (2013) S267

15 L. Garrison and G. L. Kulcinski, Phys. Scr. T159 (2014) 014020

16 H. Greuner et al., J. Nucl. Mater. 367-370 (2007) 1444

17 H. Maier et al., J. Nucl. Mater. 438 (2013) S921

18 H. Maier et al., Phys. Scr. T159 (2014) 014019

19 G. Sergienko et al., J. Nucl. Mater. 363-365 (2007) 96

20 W. H. Press, Numerical Recipes, third edition, Cambridge University Press, Cambridge 2007, chapter 15

21 R. Behrisch, W. Eckstein (Eds.), Sputtering by Particle Bombardment, Springer Berlin 2007; Topics in Applied Physics 110

22 V. A. Semenov et al., Phys. of the Solid State 56 (2014) 29

23 M Gryzinski, Phys. Rev. 138 (2A) (1965) 305

24 E. Hulpke and J. Lüdecke, Phys. Rev. Lett. 68 (1992) 2846 